\title{
Lung cancer in patients with idiopathic pulmonary fibrosis
}

\author{
J. Park*, D.S. Kim*, T.S. Shim*, C-M. Lim*, Y. Koh*, S.D. Lee*, W.S. Kim*, W.D. Kim*, J.S. Lee*, \\ K.S. Song"
}

Lung cancer in patients with idiopathic pulmonary fibrosis. J. Park, D.S. Kim, T.S Shim, C-M. Lim, Y. Koh, S.D. Lee, W.S. Kim, W.D. Kim, J.S. Lee, K.S. Song. (C) ERS Journals Ltd 2001.

ABSTRACT: Idiopathic pulmonary fibrosis (IPF) was reported to be associated with increased risk of lung cancer as a result of the occurrence of atypical or dysplastic epithelial changes in fibrosis which progressed to invasive malignancy. In that situation, the cancer will develop in the area of major fibrosis. To investigate the direct relationship between fibrosis and cancer development, the real concordance rate of the two lesions in the chest computed tomography (CT) was analysed and compared to the histological types of lung cancer.

The subjects included 63 patients with combined lung cancer and IPF (IPF-CA), 218 patients with lone IPF, and 2,660 patients with primary lung cancer. All patients were diagnosed at Asan Medical Center during the same period.

The age, percentage of smokers, and the male sex were significantly higher in IPFCA compared with lone IPF. The odds ratio of smoking was 2.71 compared to nonsmoking IPF controls. In IPF-CA, 56\% of the cancer was located in the periphery of the lung and $52 \%$ in the upper lobe. The majority of the cancers $(64 \%)$ were found in the nonfibrotic area at chest CT. The most frequent cell type was squamous cell carcinoma $(35 \%)$, and there was no significant difference in the cancer cell type between IPF-CA and total lung cancer population.

These findings suggest that in combined lung cancer and idiopathic pulmonary fibrosis patients, the features of the lung cancer are similar to the total lung cancer population.

Eur Respir J 2001; 17: 1216-1219. *Dept of Pulmonary Medicine and
\#Diagnostic Radiology, Asan Medical
Center, School of Medicine, University
of Ulsan, Seoul, Korea.

Correspondence: D.S. Kim, Division of Pulmonology, Dept of Internal Medicine, Asan Medical Center, School of Medicine, University of Ulsan, 388-1 Pungnap-dong, Songpa-gu, Seoul, Korea (Zip) $138-736$.

Fax: 82222246968

Keywords: Chest computed tomography

concordance with fibrosis

histological type

idiopathic pulmonary fibrosis

lung cancer

risk factor

Received: May 231999

Accepted after revision December 29 2000
Idiopathic pulmonary fibrosis (IPF) was thought to be associated with increased risk of lung cancer on the basis of two reasons. Firstly, autopsy studies showed that lung cancer was found simultaneously in patients with IPF [1]. Secondly, there were several epidemiological reports of increased incidence of lung cancer during the follow-up of IPF [2-6]. Recently, HUBBARD et al. [7] reported increased incidence of lung cancer in IPF patients compared to age- and sex-matched control population in a large population-based cohort study. However, not all studies have shown an increased risk of lung cancer in IPF [8, 9]. Assuming that IPF is associated with an increased risk of lung cancer, the mechanism is not certain. Because cigarette smoking itself is also an independent risk factor for IPF [10], smoking can be a confounding factor for the association of both diseases. There may also be other confounding factors for both conditions, like unknown occupational or environmental exposure $[11,12]$. Another possibility is that fibrosis itself may contribute to the development of cancer. It was proposed that fibrosis might be involved in the carcinogenesis by the occurrence of atypical or dysplastic epithelial changes in pulmonary fibrosis which progressed to invasive malignancy $[1,13,14]$. In that situation, the cancer will develop in the region not far from the area of major fibrosis. However, there is no study focusing on the location of the cancer in relation to the fibrotic lesion. Nowadays, the concordance of two lesions can be easily examined by chest computed tomography (CT). Also, it may be helpful to know whether the histological types of the cancer in IPF are different from the cancer without fibrosis. This study was performed to investigate whether the fibrosis is directly related to cancer development by the analysis of the real concordance rate of the lesions on the chest $\mathrm{CT}$, and to compare the histological types of lung cancer in 63 patients with lung cancer and IPF.

\section{Material and methods}

\section{Study subjects and method}

This retrospective study was performed at Asan Medical Center in Seoul, Korea, which is a university affiliated tertiary referral centre of 2,000 bed-size. All the patients diagnosed as IPF and lung cancer from May, 1989 to August, 1998 were enrolled in the study.

IPF was defined as: 1) diffuse interstitial lung disease without known aetiology such as drug toxicity, environmental exposures, or collagen vascular diseases; 
2) compatible clinical findings such as inspiratory crackles (velcro rale) in both lower lung fields; and 3) pathological confirmation by surgical (open or thoracoscopic) lung biopsy, or high-resolution computed tomography (HRCT) showing typical patterns of IPF such as subpleural reticular densities or honeycombing lesions predominantly in lower lobes [15-18]. Any patients who had evidence of localized or focal pulmonary fibrosis by radiography were excluded from the patients of the primary lung cancer group. During the study period, 281 patients were diagnosed as IPF and 2,660 patients as primary lung cancer at the same institute. Surgical lung biopsy for the diagnosis of IPF was performed in 91 patients. Among 281 patients with IPF, 63 patients $(22 \%)$ had combined lung cancers and idiopathic pulmonary fibrosis (IPF-CA). Central lung cancer was defined as cancer with an endobronchial lesion seen by bronchoscopy or with a location $>3 \mathrm{~cm}$ from visceral pleura [19].

\section{Methods}

A retrospective analysis of histological types of the cancer and the risk factors of lung cancer such as age, sex, smoking, and occupational history were performed. All the data were obtained from the medical records. Detailed smoking history, including total pack-yrs of smoking, were obtained from the medical records for all except five patients. On all 63 IPF-CA patients the chest CT was re-examined and the patients were divided into two groups according to the location of the cancer and the fibrotic lesion, Group 1: the cancer was located inside the fibrotic area (F-CA), and Group 2: the cancer developed in the nonfibrotic area (NF-CA).

\section{Statistical analysis}

All values are given as mean $\pm \mathrm{SD}$. Chi-squared tests and Fisher's exact tests were used in categorical data and unpaired t-tests were used in continuous data. Multivariate analysis was performed using the logistic regression method. $\mathrm{p}$-Value $<0.05$ was considered to be statistically significant.

\section{Results}

Compared to the lone IPF patients, age, male sex, and proportion of smokers were significantly higher in IPF-CA by univariate (table 1) and multivariate analysis (table 2). Odds ratio of smoking for the

Table 1.-Demographic feature of the subjects

\begin{tabular}{lccr}
\hline & Lone IPF & $\begin{array}{c}\text { IPF with } \\
\text { lung cancer }\end{array}$ & p-value \\
\hline Subjects n & 218 & 63 & \\
Age yr & $62.4 \pm 11.8$ & $66.8 \pm 7.8$ & 0.006 \\
Sex M:F (\% M) & $129: 89(59)$ & $61: 2(97)$ & $<0.001$ \\
Smoker \% & 59 & 89 & $<0.001$ \\
Smoking pack-yrs & $21.3 \pm 22.2$ & $41.5 \pm 22.1$ & $<0.001$ \\
\hline
\end{tabular}

IPF: idiopathic pulmonary fibrosis; M: male; F: female.
Table 2. - Multivariate analysis about development of lung cancer in total idiopathic pulmonary fibrosis group

\begin{tabular}{lrc}
\hline & OR $(95 \% \mathrm{CI})$ & p-value \\
\hline Age $\geqslant 60$ yrs & $2.41(1.10-5.25)$ & 0.026 \\
Sex (male) & $11.04(2.46-49.6)$ & 0.002 \\
Smoker & $2.71(1.91-6.21)$ & 0.018 \\
\hline
\end{tabular}

OR: odds ratio; $95 \% \mathrm{CI}$ : 95\% confidence interval.

development of lung cancer was 2.71. Furthermore, compared to the total lung cancer group, the age, male sex, and proportion of smokers were also significantly higher in IPF-CA (table 3). In the IPF-CA group, squamous cell carcinoma was the most frequent cell type $(35 \%)$, followed by adenocarcinoma, small cell carcinoma, and large cell carcinoma. In one doubleprimary cancer, both lesions (small cell and squamous cell carcinoma) were detected at the same time with IPF and located in the nonfibrotic area. There was no significant difference in the distribution of cell types between the total lung cancer group and IPF-CA group (table 3 ). In contrast to previous reports, more cancers were found in the upper lung, i.e. $20(31 \%)$ in right upper lobe, $13(20 \%)$ in left upper lobe, $2(3 \%)$ in right middle lobe, $19(30 \%)$ in right lower lobe, and $8(13 \%)$ in left lower lobe. Among the 63 IPF-CA, 40 patients $(56 \%)$ had the cancers located in the periphery of the lung. In the peripheral lung cancer group, only 23 cancers ( $37 \%$ of all IPF-CA, $66 \%$ of peripheral cancer) showed as F-CA on the chest CT (table 4). There was a higher tendency to develop adenocarcinoma (44\%) in the F-CA compared to the NF-CA $(23 \%)$ group, but the difference was not statistically significant $(p=0.081)$. All the small cell carcinomas were located centrally and of the NF-CA type (table 4). There were no significant differences in the stage of lung cancer, smoking history, age and sex between F-CA and NF-CA groups.

\section{Discussion}

The presented data show that despite the tendency for peripheral location of the cancer in IPF-CA, the

Table 3. - Comparison between total lung cancer and lung cancer with idiopathic pulmonary fibrosis (IPF) combined lung cancer with idiopathic pulmonary fibrosis (IPF-CA) groups

Total lung cancer IPF-CA p-value

\begin{tabular}{lccl}
\hline Subjects n & 2660 & 63 & \\
Age yr & $61.3 \pm 10.7$ & $66.8 \pm 7.8$ & 0.0001 \\
Smoker \% & 75.6 & 88.9 & 0.002 \\
Smoking pack-yrs & $30.9 \pm 21.6$ & $41.5 \pm 22.1$ & 0.001 \\
Sex M:F (\% M) & $2124: 536(80)$ & $61: 2(97)$ & 0.001 \\
Histological type & & & \\
$\quad$ Squamous cell & $1188(45)$ & $22(35)$ & 0.151 \\
Adenocarcinoma & $909(34)$ & $19(30)$ & 0.562 \\
Large cell & $40(2)$ & $4(6)$ & 0.018 \\
Small cell & $390(15)$ & $12(19)$ & 0.303 \\
Others & $133(5)$ & $6(10)$ & \\
\hline
\end{tabular}

Data presented as n (\%) unless otherwise stated. M: male; F: female. 
Table 4.-Comparison of the patients according to the location of lung cancer

\begin{tabular}{lccc}
\hline & \multicolumn{2}{c}{ Lung cancer } & \multirow{2}{*}{ p-value } \\
\cline { 2 - 3 } & $\begin{array}{c}\text { Fibrotic } \\
\text { area }\end{array}$ & $\begin{array}{c}\text { Nonfibrotic } \\
\text { area }\end{array}$ & \\
\hline Subjects n & $23(37)$ & $40(64)$ & \\
Age yr $^{+}$ & $66.5 \pm 8.4$ & $66.9 \pm 7.6$ & 0.846 \\
Sex M:F \% M & $22: 1(96)$ & $39: 1(98)$ & 1.00 \\
Smoker \% & 83 & 93 & 0.247 \\
Smoking pack-yrs & $40.5 \pm 29.0$ & $42.1 \pm 17.4$ & 0.826 \\
Histology & $9(39)$ & $13(33)$ & 0.645 \\
Squamous cell & $10(44)$ & $9(23)$ & 0.081 \\
Adenocarcinoma & $3(13)$ & $1(33)$ & \\
Large cell & $1(0)$ & $4(10)$ & \\
Not specified & $0(0)$ & $12(30)$ & 0.002 \\
Small cell & & $1(33)$ & \\
Multiple lung & & & \\
$\quad$ cancer & $2(9)$ & $4(15)$ & \\
Stage & 0 & $2(7)$ & \\
I & $7(33)$ & $4(15)$ & \\
II & $7(33)$ & $12(44)$ & \\
IIIa & $6(27)$ & $5(19)$ & \\
IIIb & & & \\
IV & & &
\end{tabular}

Data presented as n (\%) unless otherwise stated. M: male; F: female; ${ }^{+}$: mean \pm SD.

real concordance rate of lung cancer with a fibrotic lesion in IPF was low (37\%). Furthermore, the most frequent histological type of IPF-CA was squamous cell carcinoma, and age, male sex, and smoking were risk factors for lung cancer in IPF, similar to lung cancer in the general population.

In previous literature, IPF-CA was reported to be more frequent in the periphery of the lung and the lower lobe, where the fibrosis was predominant, suggesting a direct relationship [5, 6, 19]. The peripheral location of the lung cancer was reported as $88 \%$ by Nagai and Chiyotani [5], $91 \%$ by Mizushima and Kobayashi [2] and $66 \%$ by Lee et al. [19]. If fibrosis is directly related to the development of the cancer, cancer will develop inside or in close proximity to the area of major fibrosis. Having examined the real concordance of the cancer and the fibrosis by chest CT it was found that only $37 \%$ of lung cancers $(66 \%$ of peripheral cancer) were located inside the fibrotic area. The frequency of the peripheral cancer in IPF-CA was $55 \%$ in the present study, which was lower than previously reported. The reason for this discrepancy was not certain, but there is a possibility that IPF was detected early by the cancer work-up (including chest CT) in this study's patients, especially in whom IPF was diagnosed at the same time as the cancer (concurrent detection group). In order to avoid bias, the frequency of peripheral and central cancer was compared between two groups (concurrent detection group and nonconcurrent detection group). No significant difference was found between these two groups ( $51 \%$ in concurrent group, $67 \%$ in nonconcurrent group), suggesting that the low frequency of peripheral cancer was not due to concurrent detection. Additionally, no significant differences were found in other parameters such as histological types, stage of the lung cancer or the concordance rate of cancer and fibrosis (33\% in concurrent, $44 \%$ in nonconcurrent detection groups) between the two groups. Another possibility of bias was a misdiagnosis of usual interstitial pneumonia, because open or thoracoscopic lung biopsy was done in only one-third of the IPF patients. However, all of the patients had compatible clinical features and typical HRCT findings, which were proposed as diagnostic criteria of IPF without surgical lung biopsy by the American Thoracic Society/European Respiratory Society consensus statement [20].

There have been controversies on the most frequent cell types of the lung cancer in IPF-CA. Initially, adenocarcinoma was reported as the most frequent histological type, and some authors [6, 13] suggested that high frequency of adenocarcinoma in IPF-CA was related to the fibrotic scars. Other studies [3, 5, 19] however, showed that squamous cell carcinoma was the most frequent type. The presented study also found that squamous cell carcinoma was the most common type and the distribution of the histological types was similar to general lung cancer. The exception was small cell carcinoma, which is a central disease, and all the small cell carcinomas were located in the nonfibrotic area. Even though not statistically significant, there was a tendency for more adenocarcinoma in the F-CA group. However, this can be explained by the fact that adenocarcinoma is the most frequent cell type in peripheral lung cancers in general, and the fibrosis of IPF is characteristically present in the periphery of the lung.

Because smoking is a known risk factor for the development of IPF, it can be a serious confounding factor for IPF and lung cancer. Some of the previous studies have shown that smoking increased the risk of lung cancer even in patients with $\operatorname{IPF}[3,5,6]$. The presented data suggest that not only smoking, but also the male sex was a strong risk factor for lung cancer in patients with IPF. The odds ratio however, was unstable due to the small number of female patients with IPF-CA (only two patients). In Korea, smokers are predominantly male. According to the reports of the National Statistical Office of Korea in 1995, 62\% of males were smokers compared to only $6 \%$ of females. Since most of the effect of male sex in the presented study may actually be due to smoking, this can explain the relatively low odds ratio for the smoking data ( 5.6 by univariate analysis and 2.71 by multivariate analysis). Other limitations in the present study include the following: total numbers of subjects were relatively small, and there is a possibility of incorrect information, because all the data were obtained from the medical records, even though the detailed smoking history was available for $98 \%$ of the subjects. The remote exposures to possible dusts which might cause both pulmonary fibrosis and lung cancer $[10-12]$, an inherent limitation of all retrospective studies, could not be completely excluded in the subjects.

Despite these limitations, the results presented suggest that the features of lung cancer in idiopathic pulmonary fibrosis are similar to general lung cancer. However, further prospective controlled study using 
large numbers of pathologically proven idiopathic pulmonary fibrosis patients is required.

\section{References}

1. Huddad R, Massaro D. Idiopathic diffuse interstitial pulmonary fibrosis (fibrosing alveolitis), atypical epithelial proliferation and lung cancer. $\mathrm{Am} \mathrm{J} \mathrm{Med}$ 1968; 45: $211-219$.

2. Mizushima Y, Kobayashi M. Clinical characteristics of synchronous multiple lung cancer associated with idiopathic pulmonary fibrosis. Chest 1995; 108: 1272 1277.

3. Turner-Warwick M, Lebowitz M, Burrows B, Johnston A. Cryptogenic fibrosing alveolitis and lung cancer. Thorax 1980; 35: 496-499.

4. Kinoshita A, Hirose K, Taniguchi T. Lung cancer associated with idiopathic interstitial pneumonia. Jpn J Thorac Dis 1990; 28: 852-858.

5. Nagai A, Chiyotani L. Lung cancers in patients with idiopathic pulmonary fibrosis. Tohoku J Exp Med 1992; 167: $231-237$

6. Kawai T, Yakumaru K, Suzukiet M, Kageyama K. Diffuse interstitial pulmonary fibrosis and lung cancer. Acta Pathol Jpn 1987; 37: 11-19.

7. Hubbard R, Venn A, Lewis S, Britton J. Lung cancer and cryptogenic fibrosing alveolitis. A populationbased cohort study. Am J Respir Crit Care Med 2000; 161: $5-8$.

8. Harris JM, Cullinan P, McDonald JC. Does cryptogenic fibrosing alveolitis carry an increased risk of death from lung cancer? $J$ Epidemiol Community Health 1998; 52: 602-603.

9. Wells C, Mannino DM. Pulmonary fibrosis and lung cancer in the United States: Analysis of the multiple cause of death mortality data, 1979 through 1991. South Med J 1996; 89: 505-510.
10. Baumgartner KB, Samet JM, Stidley CA, et al. Cigarette smoking - a risk factor for idiopathic pulmonary fibrosis. Am J Respir Crit Care Med 1997; 155: $242-248$.

11. Hubbard R, Lewis S, Richards K, Johanston I, Britton J. Occupational exposure to metal or wood dust and aetiology of cryptogenic fibrosing alveolitis. Lancet 1996; 347: 284-289.

12. Iwai K, Mori T, Yamada N, Yamaguchi M, Hosoda Y. Idiopathic pulmonary fibrosis: epidemiologic approaches to occupational exposure. Am J Respir Crit Care Med 1994; 150: 670-675.

13. Meyer EC, Liebow AA. Relationship of interstitial pneumonia honeycombing and atypical epithelial proliferation to lung cancer. Cancer 1965; 18: $322-$ 351.

14. Fraire AE, Greenberg SD. Carcinoma and diffuse interstitial fibrosis of lung. Cancer 1973; 31: $1078-$ 1086.

15. Cherniack RM, Crystal RG, Kalica AR. Current concepts in idiopathic pulmonary fibrosis: a road map for the future. Am Rev Respir Dis 1991; 143: $680-$ 683.

16. Raghu G. Interstitial lung disease: a diagnostic approach. Are CT scan and lung biopsy indicated in every patient? Am J Respir Crit Care Med 1995; 151: 909-914.

17. King TE. Diagnostic advances in idiopathic pulmonary fibrosis. Chest 1991; 100: $238-241$.

18. Marinelli WA. Idiopathic pulmonary fibrosis: progress and challenge. Chest 1995; 108: 297-298.

19. Lee HJ, Im JG, Ahn JM, Yeon KM. Lung cancer in patients with idiopathic pulmonary fibrosis. J Comput Assist Tomog 1996; 20: 979-982.

20. American Thoracic Society. Idiopathic pulmonary fibrosis: Diagnosis and treatment. International Consensus Statement. Am J Respir Crit Care Med 2000; 161: $646-664$. 